\title{
Factors Influencing Stages of Change for Contraceptive Use in College Students : A Path Analysis
}

\author{
Myung Sook Choi ${ }^{1}$, Hyunjeong Shin ${ }^{2, *}$ \\ ${ }^{1}$ Department of Nursing, Seoil University, South Korea \\ ${ }^{2}$ College of Nursing, Korea University, South Korea \\ *Corresponding Author: hyunjshin@korea.ac.kr
}

Copyright (C) 2015 Horizon Research Publishing. All rights reserved.

\begin{abstract}
Objectives: The purpose of this study was to examine the relationships of self-efficacy, social influences, decisional balance, consistency in past contraceptive use, and stages of change for contraceptive use in college students. Study design: A cross-sectional correlational study was conducted on a convenience sample of 185 Korean college students. Data collection was conducted by three trained research assistants over a six-month period in 2013. Path analysis with AMOS software was used to identify the effects of predictor variables on stages of change for contraceptive use. Results: Social influences, consistency in past contraception use, and self-efficacy had a direct effect on the stages of change for contraception use. Although decisional balance did not show a direct effect, it had a significant indirect effect on the stages of change through self-efficacy. These predicting variables explained $40 \%$ of the variance of stages of change for contraception use. Conclusions: The results suggested that the TTM is useful in explaining college students' contraceptive behavior and that self-efficacy partially mediated the role of cognitive (social influence) and behavioral (past contraception use) factors on the stages of change for contraceptive use.
\end{abstract}

Keywords Contraception, Transtheoretical Model, College Students, Stage of Change

\section{Introduction}

College students are generally a sexually active group, as most of them have already engaged in intercourse in their lifetimes and more than two thirds of them report current sexual activity [1-2]. Nonetheless, about 1 in 5 sexually active students does not use contraception or uses traditional methods such as periodic abstinence and withdrawal [1-3]. This may result in negative social and physical consequences including unwanted pregnancy and abortion. Studies from several countries including Turkey, Korea, and the U.S. have shown that $5-22 \%$ of college students who have had intercourse had the experience of unwanted pregnancy or got someone pregnant unintentionally [2-5].

A variety of factors have been reported to influence contraceptive use among this population [6-9]. However, how these factors are associated with each other, and how they influence the stages of behavioral change for contraceptive use are not clear, warranting more research. The current study tested hypothesized relationships between predicting variables of the stages of change for contraceptive use, guided by the transtheoretical model (TTM).

\subsection{Factors Affecting Stages of Change for Contraceptive Use}

Several previous studies support the relationships between contraceptive use and the predicting variables. The relationship between stages of change for contraceptive use and self-efficacy has been demonstrated in cross-sectional studies [10]. Men's self-efficacy for contraception is a significant predictor of their stages of change to accept intrauterine devices (IUD) for contraception [7]. Findings from qualitative studies with women indicated that a high degree of self-efficacy for contraceptive use may contribute to successful initiation and continuation of contraceptive use [11]. Self-efficacy was also reported to contribute to the use of condom and oral contraceptives [12-13].

Decisional balance also influences stages of change for contraceptive use. Sound decisions for health behaviors involve careful consideration of all pertinent information into a decisional matrix of comparative benefits and disadvantages [10]. Both positive and negative aspects of contraceptive use are related to stages of change for contraceptive and condom use [10]. Another previous study reported that decisional balance for contraceptive use influenced condom use through motivational readiness [8]. Decisional balance was also reported to predict readiness to accept IUDs; in a study among men, the group with higher motivational readiness for IUD use showed higher scores of pros and lower scores of cons for using IUD than the group with lower motivational readiness [7]. Dempsey et al. [6], however, reported in their study of oral contraceptive continuation, that women who considered both its 
advantages and disadvantages important were more likely to continue to use it.

Social influences are individual perceptions of the social pressures put on individuals to conduct or not conduct behaviors [14]. The perception of peers' or significant others' behaviors and recommendations can be considered important for the contraceptive behavior in young people [14]. Findings from a review of qualitative research noted that social networks including friends, parents, and schools influence views regarding the use of contraceptives [15]. The relationship between social influences and contraceptive use, however, can be mediated by self-efficacy and decisional balance [9]. A study with young people showed that the decision-making processes for contraceptive use were affected by social influences including social network experiences and recommendations of contraceptive use [16]. Outlaw et al. [8] also reported significant indirect effects from social influences to condom use through decisional balance. Studies among Taiwanese people noted that social influences affect contraceptive intention indirectly through self-efficacy and decisional balance $[9,14]$.

Past contraceptive experiences have repeatedly demonstrated to be an influencing factor on contraceptive use. A previous study among Chinese university students showed that condom use during the first sexual experience and oral contraceptive use in the previous year were significant predictors for current condom use [12]. Wang et al. [9] also reported that past contraceptive use has significant direct effects on both self-efficacy and current contraceptive use. Prior contraceptive experience may affect the decision-making process for current or future contraceptive use and the next choice for contraceptive options [16].

Previous research suggests that the variables of self-efficacy for contraceptive use, social influences, decisional balance, and past contraceptive experiences influence current contraceptive use. However, most previous contraceptive studies focused on the use of specific contraceptives and their predictors, disregarding the stages of change individuals pass through in modifying their contraceptive behaviors. In addition, none of the studies have described these variables simultaneously and tested the mediating effects of them on stages of change for contraceptive use. Additional research is needed to explore these inter-relationships and to advance the existing body of knowledge.

\subsection{Conceptual Framework}

In the current study, the TTM [17] was used to formulate hypothesized relationships between stages of change for contraceptive use and the affecting variables. The TTM describes an individual's behavioral progress through the stages [17]. The core constructs of the TTM include stages of change, self-efficacy, and decisional balance [18].

Stages of change is the central construct of the TTM involving progress through a series of five stages: (a) precontemplation: the person has no intention to take action in the next 6 months, (b) contemplation: the person intends to change in the next 6 months, (c) preparation: the person intends to take action in the next month, (d) action: the person has changed his or her behavior within the past 6 months, and (e) maintenance: the person maintains the behavior for more than 6 months [18]. Self-efficacy reflects the person's confidence to overcome barriers for engaging in the health behavior. High self-efficacy is associated with late stages of change [7, 19-20]. Decisional balance reflects on the person's relative weighing of the pros and cons of changing and represents cognitive and motivational aspects of human decision-making [18]. When the individual perceives benefits to be greater than barriers, a greater possibility of performing that behavior exists [9]. Previous studies reported that people in the maintenance stage of the TTM showed higher levels of perceived benefits and lower levels of perceived barriers than people in the precontemplation stage [21-22].

For the current study, the theoretical framework of contraceptive use behavior from Wang et al.'s [9] study was used. In addition to the three core constructs of the TTM, social influences and past contraceptive use were included in the model. Social influences are defined as perceptions of social support and pressure for contraception use [9]. Wang et al. [9] showed that social influences had direct effects on both self-efficacy and decisional balance for contraceptive use in their study. Using the concepts of stages of change, decisional balance, and self-efficacy from the TTM and empirical evidence on social influences and past contraception, a model for contraception use behavior was constructed (Figure 1).

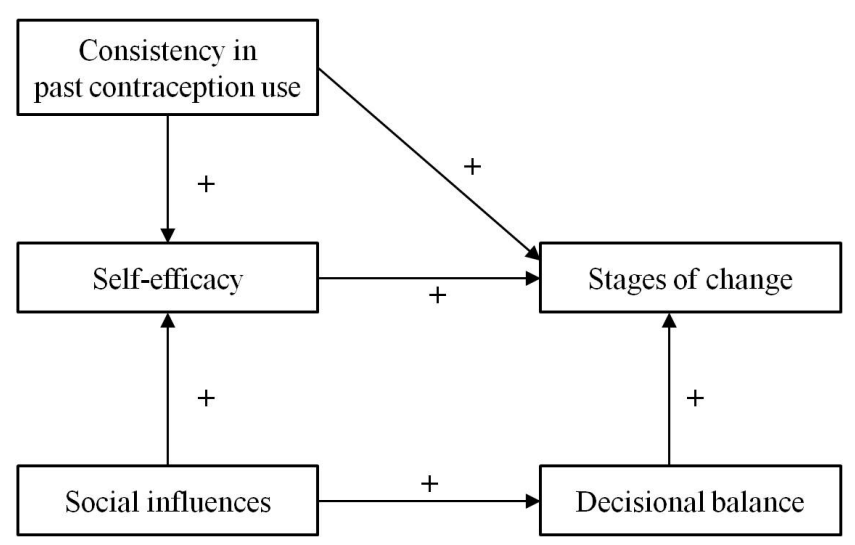

Figure 1. Hypothesized model

\subsection{Purpose}

The purpose of this study was to examine the relationships of self-efficacy, social influences, decisional balance, consistency in past contraceptive use, and stages of change for contraceptive use in college students. We hypothesized that higher self-efficacy, consistency in contraceptive use, and decisional balance would be associated with higher levels of stages of change for contraceptive use. Also, 
positive relationships were posited between social influences and both self-efficacy and decisional balance. Furthermore, it was hypothesized that self-efficacy would mediate the effects of consistency in past contraceptive use on the stages of change for contraceptive use.

\section{Materials and Methods}

\subsection{Study Design}

A cross-sectional correlational design was used in this study.

\subsection{Sample and Data Collection}

Data collection was conducted by three trained research assistants over a six-month period in 2013. Data were collected from a convenience sample of students in a variety of grades at three universities located in Korea. Three trained research assistants went to random classrooms and advertised the study. Of the 315 students who showed an interest in the study, 15 students finally refused to participate. The questionnaires were self- administered in a classroom. Of the 300 participants enrolled in the study, 12 did not complete the baseline data collection and were removed from this study. Of the 288 participants who were screened for the study, 103 students were not eligible due to having no sexual partner during past year. The current study sample consisted of 185 participants.

In the current study, we performed correlation analysis, one-way ANOVA, and path analysis for statistical analysis. A power analysis for correlation analyses (two-tailed) with an alpha of .01 , a medium effect size $(r=.30)$, and a power of .90 indicated that 157 subjects were the appropriate sample for this study [23]. A power analysis for one-way ANOVA with five groups, an alpha of .05, a medium effect size $(f=0.30)$, and a power of .80 indicated that 160 would be required (Cohen et al., 2003). For path analysis, 10 cases per variable can be considered suitable because it is based on regression technique [24]. Thus, a minimum sample size of 50 was required ( 5 variables $\times 10$ cases). Thus, the sample size of 185 was sufficient for all analyses.

Approval for the study was obtained through the institutional review board and ethical committee. Students who agreed to participate in the study signed the informed consent form. To ensure privacy, they were required not to sit too close to each other. Questionnaire responses were anonymous.

\subsection{Measures}

\subsubsection{Self-efficacy for Contraceptive Use}

The self-efficacy measure for contraception use was adapted from another study [25]. Since it was developed specifically for condom use, we modified some items for general contraception use (e.g., The item When my partner dislikes condom use, I can resist sexual intercourse was changed to When my partner does not want to use some form of birth control, I can resist sexual intercourse). The scale consisted of 10 items rated on a 5-point Likert scale ranging from "not at all" to "extremely". Higher scores indicate greater self-efficacy in one's ability to resist temptation and to use contraception. Cronbach's alpha coefficient for the 10 items was .75 in this study.

\subsubsection{Social Influences}

A 4-item scale was developed by the authors to evaluate social influences on contraception use from significant others: parents, teachers, sexual partners, and peers. An example of an item used is: My schoolteachers always said I should use a type of contraceptive method when having premarital sex. The items were rated on a 5-point Likert scale ranging from "not at all" to "extremely". Higher scores indicate participants perceive that significant others are more supportive of their contraception use. Cronbach's alpha coefficient for the four items was .71.

\subsubsection{Decisional Balance for Contraception}

Decisional balance was measured using the scale developed by Grimley et al. [10]. The original scale consists of two distinct sections: pregnancy prevention and disease prevention (condom use). In the current study, the pregnancy prevention scale was used. It contained 10 items of advantages in contraception use (PROS) and 10 items of disadvantages of contraception use (CONS). The scale was translated into Korean by one of the researchers and then back-translation was performed by another researcher. Following back-translation, discordant opinions were discussed and awkward expressions were revised. Participants were asked to rate how important each statement was regarding their decision whether or not to use contraceptives. The items are rated on a 5-point Likert scale ranging from " $1=$ not important" to "5 = extremely important". The balance score was computed as PROS minus CONS. Higher scores represent more PROS for contraception use. In the current study, Cronbach's alpha coefficients for the PROS and CONS were .90 and .92, respectively.

\subsubsection{Stages of Change}

The item from Grimley et al.'s [10] study was used to measure the stages of change for contraception use. It classifies individuals into one of the five stages of change for separate contraceptive behaviors: precontemplation $(1=$ not intending to use in next 6 months $)$, contemplation $(2=$ intending to accept in 6 months), preparation $(3=$ planning to accept in next 30 days), action ( 4 = using some form of contraception less than 6 months $)$, and maintenance $(5=$ using some form of contraception for 6 months and longer). Participants were asked to select the one that best represented his/her current contraceptive behavior. 
Table 1. Descriptive statistics and correlations among the study variables $(n=185)$

\begin{tabular}{|c|c|c|c|c|c|c|c|c|}
\hline Variables & $\begin{array}{c}\text { Possible } \\
\text { range }\end{array}$ & Mean & $\mathrm{SD}$ & 1 & 2 & 3 & 4 & 5 \\
\hline 1. Social influences & $4-20$ & 14.54 & 3.05 & 1.00 & & & & \\
\hline 2. Decisional balance & $-40 \sim 40$ & 14.28 & 12.29 & $.32 * *$ & 1.00 & & & \\
\hline $\begin{array}{l}\text { 3. Consistency in past } \\
\text { contraception use }\end{array}$ & $1-5$ & 3.82 & 1.03 & $.37 * *$ & $.34 * *$ & 1.00 & & \\
\hline 4. Self-efficacy & $10-50$ & 34.47 & 6.02 & $.38^{* *}$ & $.44 * *$ & $.47 * *$ & 1.00 & \\
\hline 5. Stages of change & $1-5$ & 3.78 & 1.31 & $.29^{* *}$ & $.37 * *$ & $.60^{* *}$ & $.35^{* *}$ & 1.00 \\
\hline
\end{tabular}

\subsubsection{Consistency in Past Contraceptive Use}

One item (How consistently did you use contraceptives when you had sex?) was used to measure participants' consistency in contraception use during past one year.

Responses were rated as $1=$ never used, $2=$ less than $50 \%$ of intercourse, $3=$ about $50 \%$ of intercourse, $4=$ more than $50 \%$ of intercourse, and $5=$ always used. Higher score indicates better contraception use during the past one year.

\subsection{Data Analysis}

Data analysis was conducted using SPSS program (Version 21.0). Descriptive statistics and correlation coefficients between study variables were examined. Differences across stages of change by decisional balance and self-efficacy were examined using one-way analysis of variance (ANOVA).

Path analysis with AMOS software (Version 21.0) was used to identify the effects of predictor variables on stages of change for contraceptive use. Before path analysis, multicollinearity was examined in the correlations among independent variables and was not found in the current study (range of variance inflation: 1-4; desired value for path analysis $<10$ ). Model fit indices were examined to test the overall fit of the model to the collected data including the chi-square statistic (desired $p$-value $>.05$ ) with degrees of freedom, the normed fit index (NFI; desired value $>0.90$ ), incremental fit index (IFI; desired value $>0.90$ ), comparative fit index (CFI; desired value $>0.90$ ), root mean square error of approximation (RMSEA; desired value < 0.08), and Akaike's information criterion (AIC; the smaller the AIC, the better the fit).

\section{Results}

\subsection{Characteristics of the Participants}

The mean age of participants was 22.33 years $(S D=1.82$, range: 19-29). About two thirds of them (60.5\%) were male students. About half of the participants (51.4\%) were sophomores. Most $(96.8 \%)$ were not married. More than $80 \%$ of the participants drank alcohol less than two times a week. About two thirds of them $(65.9 \%)$ were non-smokers. About one-fifth experienced their own or their partners' unintended pregnancy (21.1\%) and abortion (18.4\%). Most of the participants who reported using contraception (95.1\%) were currently using condoms. A small portion was using oral contraceptives $(2.5 \%)$ or traditional methods such as periodic abstinence and withdrawal (2.4\%). As a result of the classification of participants into stages of change for contraception use, over half of the participants were using contraceptives $(62.1 \%)$, with $21.6 \%$ placing themselves in the action stage and $40.5 \%$ in maintenance. More than $7 \%$ of the participants stated that they had no intention of contraception.

Descriptive statistics and correlations between study variables are presented in Table1.

\subsection{Decisional Balance and Self-efficacy by Stages of Change}

The scores for PROS and CONS by stages of change are presented in Table 2. There were significant differences in the scores for decisional balance and CONS across the stages of change. Post hoc Duncan test results indicated that there were significantly higher scores for decisional balance and lower scores for CONS in the action / maintenance stages compared to the precontemplation / contemplation / preparation stages. No significant differences were found across the stages in the scores for PROS.

The findings for self-efficacy scores showed that there were significant differences across the stages of change. Post hoc Duncan test results indicated that there were significantly higher scores for self-efficacy in the action / maintenance stages compared to the precontemplation / contemplation / preparation stages. 
Table 2. Scores for decisional balance and self-efficacy by stages of change for contraception use ( $\mathrm{n}=185)$

\begin{tabular}{ccccccc}
\hline & $\begin{array}{c}\text { Precontemplation } \\
(\mathrm{n}=14)\end{array}$ & $\begin{array}{c}\text { Contemplation } \\
(\mathrm{n}=20)\end{array}$ & $\begin{array}{c}\text { Preparation } \\
(\mathrm{n}=31)\end{array}$ & $\begin{array}{c}\text { Action } \\
(\mathrm{n}=40)\end{array}$ & $\begin{array}{c}\text { Maintenance } \\
(\mathrm{n}=75)\end{array}$ & $\mathrm{F}$ \\
\cline { 2 - 5 } & Mean (SD) & Mean (SD) & Mean (SD) & Mean (SD) & Mean (SD) & \\
\hline $\begin{array}{c}\text { Decisional } \\
\text { balance }\end{array}$ & $6.33(8.78)^{\mathrm{a}}$ & $11.50(5.56)^{\mathrm{a}}$ & $8.89(10.97)^{\mathrm{a}}$ & $19.96(11.96)^{\mathrm{b}}$ & $18.45(11.26)^{\mathrm{b}}$ & $5.75^{* *}$ \\
PROS & $37.00(8.05)$ & $38.92(4.19)$ & $37.95(7.76)$ & $42.50(4.97)$ & $40.61(5.78)$ & 2.43 \\
CONS & $30.67(11.29)^{\mathrm{a}}$ & $27.67(5.79)^{\mathrm{a}}$ & $29.05(11.04)^{\mathrm{a}}$ & $22.68(8.48)^{\mathrm{b}}$ & $22.36(8.41)^{\mathrm{b}}$ & $3.53^{*}$ \\
Self-efficacy & $33.44(5.03)^{\mathrm{a}}$ & $31.46(5.98)^{\mathrm{a}}$ & $33.21(5.62)^{\mathrm{a}}$ & $35.31(5.61)^{\mathrm{b}}$ & $37.89(6.38)^{\mathrm{b}}$ & $4.37^{* *}$ \\
\hline
\end{tabular}

Note. PROS: advantages in contraception use; CONS: disadvantages of contraception use; $* \mathrm{p}<.05 ; * * \mathrm{p}<.01$; a, b: Duncan test results

\subsection{Evaluation of the Hypothesized Path Model}

The initial testing of the hypothesized path model showed that five of the six paths were statistically significant, but the model fit was not satisfactory $\left(\chi^{2}(4,185)=670.12, p<.01\right.$, $\mathrm{NFI}=0.78, \quad \mathrm{IFI}=0.78, \quad \mathrm{CFI}=0.78, \quad \mathrm{RMSEA}=0.24, \quad \mathrm{AIC}=$ 702.11). To modify the model, a nonsignificant path from decisional balance to the stages of change was removed and a path from decisional balance to self-efficacy was added based on the modification indices (greater than 10). Testing of this modified model showed improvement in the fit indices, but provided some support for more modification $\left(\chi^{2}\right.$ $(4,285)=310.18, p<.01, \mathrm{NFI}=0.90, \mathrm{IFI}=0.90, \mathrm{CFI}=0.90$, RMSEA $=0.16, A I C=342.18)$. $)$. In the final model, two paths with modification indices greater than 10 were added, as they were considered theoretically reliable: a path from social influences to stages of change and a path from social influences to consistency in past contraception use. All paths of the final model were statistically significant, and showed very good fit with the data (Figure 2). The model fit indices were as follows: $\chi^{2}(2,285)=2.08, p=.35$, NFI $=0.99$, $\mathrm{IFI}=1.00, \mathrm{CFI}=1.00, \mathrm{RMSEA}=0.01, \mathrm{AIC}=342.18$.

In the final model, social influences (total effect: 0.13 , $p$ $<.01$ ), consistency in past contraception use (total effect: $0.54, \mathrm{p}<.05$ ), and self-efficacy (total effect: $0.02, \mathrm{p}<.05$ ) had a direct effect on the stages of change for contraception use. Although decisional balance did not show a direct effect, it had a significant indirect effect on the stages of change through self-efficacy. These predicting variables explained $40 \%$ of the variance of stages of change for contraception use.

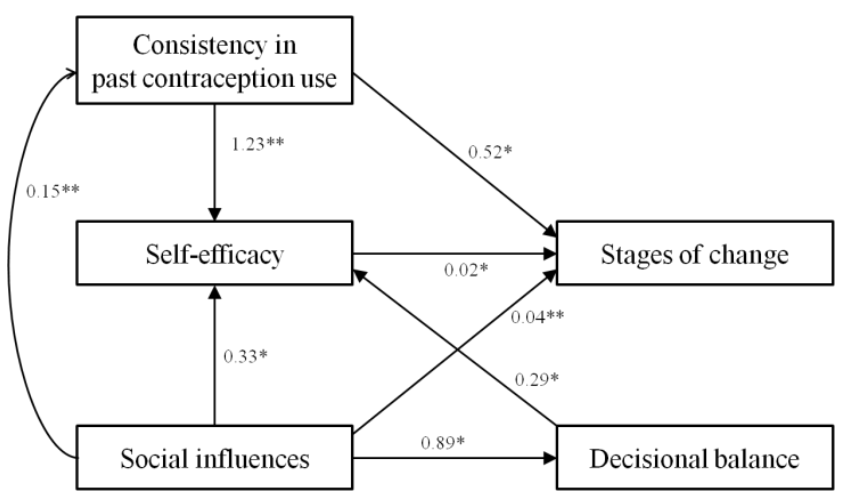

Figure 2. Final model

\section{Discussion}

Although based on small numbers, our study findings indicated that a considerable number of sexually active Korean college students have not used contraceptives; more than $35 \%$ of participants rated their position in the stages of precontemplation, contemplation, and preparation. The rate of non-users was higher than that in the previous studies from several other countries [1-3], and might lead to higher rates of unwanted pregnancy and abortion. The rate of unintended pregnancy and induced abortion tends to be higher among Asian college students than in North American or European college students $[2-4,26]$. This may be associated with the underlying culture in many Asian countries that considers talking about sexual issues and premarital sex to be taboo, which often results in insufficient information and misconceptions about contraception [3, 12]. In addition, the CONS scores of the students who were in the stages of precontemplation, contemplation, and preparation were significantly higher than those who were using contraceptives, while no significant differences were found in their PROS scores. This finding suggests that decreasing the perceptions of disadvantages in contraceptive use may be more important than increasing the perceptions of advantages for college students' contraceptive behaviors.

Among the variables, consistency in past contraceptive use had the greatest influence on the stages of change for contraceptive use directly and indirectly through self-efficacy. It suggests that students who have consistently used contraceptives during past one year may be in a higher stage of change because their past contraceptive use may increase their self-efficacy for contraception. Previous studies with young adults also reported that past contraceptive use behavior was a powerful predictor of current or future contraceptive intentions and choice showing the strongest relationship with self-efficacy $[12,27]$. The findings of past contraceptive use as a powerful predictor of intentions or stages of change for contraception has important intervention implications, suggesting that dividing young people considering their past experiences of contraceptive use would be more effective for contraception programs. People who report contraceptive use need to be encouraged to maintain their behavior, while for those who have not, health care professionals need to be focused on the factors that motivate use of contraceptives [27]. 
Social influence was the second strongest predictor of stages of change for contraception. In the current study, indirect effects of social influence were greater than the direct effect on stages of change. Furthermore, the indirect effects of social influence on stages of change were greater than total effects of either decisional balance or self-efficacy. These results indicate social influences may take precedence over personal considerations, which seems to be from East Asian culture placing a greater emphasis on norms reported by other members of the same culture [9]. Developing and providing supportive social influences from parents, school teachers, and peers should be considered an important strategy to improve contraceptive attitude and self-efficacy among sexually active young people $[9,14]$. Consistent with prior study results [8], the current study suggests that social cognitive factors (i.e., social influence, self-efficacy, and decisional balance) are associated with contraceptive behavior in college students. Interventions that promote these social cognitive factors may be useful in understanding and conceptualizing their contraceptive behaviors [15].

In this study, self-efficacy mediated the effects of social influence and consistency in past contraceptive use on stages of change for contraceptive behavior. The strong mediating influence of self-efficacy on contraceptive behaviors has been previously noted $[8-9,14]$. While supportive influences from family, teachers, and peers has important effects on college students' contraceptive behavior, the current study results show that it is the pathway through which social influence operate (i.e., self-efficacy) that is more important to stages of change for contraceptive behavior.

Although the TTM was supported by the data from a sample of college students, there are several limitations that should be considered when interpreting the results. This study used a cross-sectional design; thus, definitive conclusions about direction of causality between the relationships cannot be determined. Secondly, data were collected using a convenience sampling method from students attending three universities located in Korea and may not represent Korean college students. Third, much of variance in the stages of change for contraceptive behavior remained unexplained. There are a number of contraceptive use determinants (e.g., gender, types of contraceptive method, contraceptive knowledge and attitude, and partner relationships) that are not accounted for by the TTM [16, 27-28]. No single theory can account for all of the complexities of behavioral change [18]. Modeling contraceptive behavior including variables beyond the TTM would enhance the explanatory power for college students' contraceptive behavior. In addition, although the stage of change is a strong predictor for contraceptive behaviors, it cannot be a direct indicator of the overall proportion of behavioral change. Importantly, contraceptive behaviors cannot be performed by only individual intention, but require partner involvement and assistance in many cases [27]. Lastly, another important aspect worth mentioning is the risk of lumping all contraceptive methods and gender with no subgroup analyses. Although the aim of the study was mostly to test the proposed model, we know that different contraceptive methods have different demands on couples. For instance, if the man who is the dominant in the relationship is hesitant to use condom it would not matter much whether the woman is motivated to use contraception or not.

\section{Conclusions}

The present study verified the usefulness of TTM in understanding factors affecting contraceptive behaviors in college students. Consistent with prior study results guided by the TTM [8-9, 14], self-efficacy and decisional balance partially mediated the role of cognitive (social influence) and behavioral (past contraception use) factors on the stages of change for contraceptive use. The variables accounted for $40 \%$ of the variance in the stages of change for contraceptive use. The study findings suggest that the TTM is useful in explaining college students' contraceptive behavior.

In conclusion, current study results suggest that programs aimed at promoting contraceptive behavior in college students should seek ways and means to increase self-efficacy and to facilitate supportive communications with family or peers about contraception. Also, interventions will be more effectual and cost-effective when they are matched to the individual stages of behavioral change [10]. For instance, students who are in the action / maintenance stages need to be encouraged to maintain their contraceptive behavior, while those who are in the precontemplation stage will require messages that focus on the factors that motivate the use of contraceptives, decreasing their perceptions of the disadvantages of contraception use [27].

\section{Acknowledgements}

The present research was conducted with the Research Grant of Seoil University in 2012.

\section{REFERENCES}

[1] Huber, L. R. B., \& Ersek, J. L. (2009). Contraceptive use among sexually active university students. Journal of Women's Health, 18, 1063-1070.

[2] Trieu, S. L., Bratton, S., Marshak, H. H. (2011). Sexual and reproductive health behaviors of California community college students. Journal of American College Health, 59, 744-750.

[3] Erenel, A. S., \& Golbasi, Z. (2011). Unprotected sexual intercourse and unplanned pregnancy experience of Turkish university students. Sexuality and Disability, 29, 75-80.

[4] Buhi, E. R., Marhefka, S. L., \& Hoban, M. T. (2010). The state of the union: Sexual health disparities in a national sample of US college students. Journal of American College Health, 58, 337-346. 
[5] Sohn, A. R., \& Chun, S. S. (2005). Comparing sexual attitude, sexual initiation and sexual behavior by gender in Korean college students. Korean Association of Health and Medical Sociology, 18, 73-100.

[6] Dempsey, A. R., Johnson, S. S., \& Westhoff, C. L. (2011). Predicting oral contraceptive continuation using the transtheoretical model of health behavior change. Perspectives on Sexual and Reproductive Health, 43, 23-29.

[7] Ha, B. T. T., Jayasuriya, R., \& Owen, N. (2005). Predictors of men's acceptance of modern contraceptive practice: Study in rural Vietnam. Health Education and Behavior, 32, 738-750.

[8] Outlaw, A., Naar-King, S., Janisse, H., Parsons, J. T., \& The Adolescent Trials Network for HIV/AIDS Interventions (2010). Predictors of condom use in a multisite study of high-risk youth living with HIV. AIDS Education and Prevention, 22, 1-14.

[9] Wang, R. H., Wang, H. H., Cheng, C. P., Hsu, H. Y., \& Lin, S. Y. (2007). Testing a model of contraception use behavior among sexually active female adolescents in Taiwan. Research in Nursing and Health, 30, 628-640.

[10] Grimley, D. M., Riley, G. E., Bellis, J. M., \& Prochaska, J. O. (1993). Assessing the stages of change and decision-making for contraceptive use for the prevention of pregnancy, sexually transmitted disease, and acquired immunodeficiency syndrome. Health Education Quarterly, 20, 455-470.

[11] Suvivuo, P., Tossavainen, K., \& Kontula, O. (2009). Contraceptive use and non-use among teenage girls in a sexually motivated situation. Sex Education, 9, 355-369.

[12] Ma, Q., Ono-Kihara, M., Cong, L., Pan, X., Xu, G., Zamani, S., ... Kihara, M. (2009). Behavioral and psychosocial predictors of condom use among university students in Eastern China. AIDS Care, 21, 249-259.

[13] Peyman, N.,Hidarnia, A., Ghofranipour, F., Kazemnexhad, A., Oakley, D., Khodaee, G., \& Aminshokravi, F. (2009). Self-efficacy: Does it predict the effectiveness of contraceptive use in Iranian women? Eastern Mediterranean Health Journal, 15, 1254-1262.

[14] Wang, R. H., Cheng, C. P., \& Chou, F. H. (2008). A causal model of contraceptive intention and its gender comparison among Taiwanese sexually inexperienced adolescents. Journal of Clinical Nursing, 17, 930-939.

[15] Baxter, S., Blank, L., Guillaume, L., Squires, H., \& Payne, N. (2011). Views regarding the use of contraception amongst young people in the UK: A systematic review and thematic synthesis. The European Journal of Contraception and Reproductive Health Care, 16, 149-160.
[16] Sucato, G. S., Bhatt, S. K., Murray, P. J., \& Ott, M. A. (2011). Transdermal contraception as a model for adolescent use of new methods. Journal of Adolescent Health, 49, 357-362.

[17] Prochaska, J. O., Veliver, W. F., Rossi, J. S., Goldstein, M. G., Marcus, B. H., Rakowski, W., ... Rossi, S. R. (1994). Stages of change and decisional balance for 12 problem behaviors. Health Psychology, 13, 39-46.

[18] Prochaska, J. O., \& Velicer, W. F. (1997). The transtheoretical model of health behavior change. American Journal of Health Promotion, 12, 38-48.

[19] Plow, M. A., Finlayson, M., \& Cho, C. (2011). Correlates of stages of change for physical activity in adults with multiple sclerosis. Research in Nursing and Health, 34, 378-388.

[20] Resnick, B., \& Nigg, C. (2003). Testing a theoretical model of exercise behavior for older adults. Nursing Research, 52, 80-88.

[21] Eiser, J. R., \& Cole, N. (2002). Participation in cervical screening as a function of perceived risk, barriers and need for cognitive closure. Journal of Health Psychology, 7, 99-105.

[22] Tung, W. C. (2010). Benefits and barriers of pap smear screening: Differences in perceptions of Vietnamese American women by stage. Journal of Community Health Nursing, 27, 12-22.

[23] Cohen, J., Cohen, P., West, S. G., \& Aiken, L. S. (2003). Applied multiple regression/correlation analysis for the behavioral sciences (3rd ed.). Mahwah, NJ: Lawrence Erlbaum.

[24] Nunnally, J. C., \& Bernstein, I. H. (1994). Psychometric theory (3rd ed.). New York, NY: McGraw-Hill.

[25] Kang, H. S. (2001). Explanatory model of condom use among Korean college students. Unpublished doctoral dissertation, Yonsei University, Seoul.

[26] Virtala, A., \& Virjo, I. (2005). Consultations concerning contraception and induced abortions among university students - trends in Finland 1986-2003. Contraception, 72, 372-376.

[27] Campo, S., Askelson, N. M., Spies, E. L., \& Losch, M. (2012). Ambivalence, communication and past use" Understanding what influences women's intentions to use contraceptives. Psychology, Health \& Medicine, 17, 356-365.

[28] Peyman, N., \& Oakley, D. (2011). Married Iranian women's knowledge, attitude and sense of self-efficacy about oral contraceptives: Focus group discussion. Journal of Reproduction and Infertility, 12, 281-288. 